Article

\title{
Efficiency Enhanced Grating Coupler for Perfectly Vertical Fiber-to-Chip Coupling
}

\author{
Zan Zhang ${ }^{1, *} \mathbb{C}$, Xiaotao Shan ${ }^{1}$, Beiju Huang ${ }^{2}$, Zanyun Zhang ${ }^{3}$, Chuantong Cheng ${ }^{2}$, Bing Bai ${ }^{1}$, \\ Tianxi Gao ${ }^{1}$, Xiaobo $\mathrm{Xu}^{1}{ }^{1}$, , Lin Zhang ${ }^{1}$ and Hongda Chen ${ }^{2}$ \\ 1 School of Electronic and Control Engineering, Chang'an University, Xi'an 710064, China; \\ 2016903901@chd.edu.cn (X.S.); baibing@chd.edu.cn (B.B.); tianxigao@chd.edu.cn (T.G.); \\ xuxiaobo@chd.edu.cn (X.X.); zhanglin_dk@chd.edu.cn (L.Z.) \\ 2 Chinese Academy of Sciences, Beijing 100083, China; bjhuang@semi.ac.cn (B.H.); \\ chengchuantong@semi.ac.cn (C.C.); hdchen@semi.ac.cn (H.C.) \\ 3 School of Electronics and Information Engineering, Tianjin Polytechnic University, Tianjin 300387, China; \\ zhangzanyun@tjpu.edu.cn \\ * Correspondence: z.zhang@chd.edu.cn
}

Received: 19 April 2020; Accepted: 10 June 2020; Published: 12 June 2020

\begin{abstract}
In this work, a bidirectional grating coupler for perfectly vertical coupling is proposed. The coupling efficiency is enhanced using a silicon nitride $\left(\mathrm{Si}_{3} \mathrm{~N}_{4}\right)$ layer above a uniform grating. In the presence of $\mathrm{Si}_{3} \mathrm{~N}_{4}$ layer, the back-reflected optical power into the fiber is diminished and coupling into the waveguide is increased. Genetic algorithm (GA) is used to optimize the grating and $\mathrm{Si}_{3} \mathrm{~N}_{4}$ layer simultaneously. The optimal design obtained from GA shows that the average in-plane coupling efficiency is enhanced from about $57.5 \%(-2.5 \mathrm{~dB})$ to $68.5 \%(-1.65 \mathrm{~dB})$, meanwhile the average back-reflection in the $\mathrm{C}$ band is reduced from $17.6 \%(-7.5 \mathrm{~dB})$ to $7.4 \%(-11.3 \mathrm{~dB})$. With the help of a backside metal mirror, the average coupling efficiency and peak coupling efficiency are further increased to $87 \%(-0.6 \mathrm{~dB})$ and $89.4 \%(-0.49 \mathrm{~dB})$. The minimum feature size of the designed device is $266 \mathrm{~nm}$, which makes our design easy to fabricate through $193 \mathrm{~nm}$ deep-UV lithography and lowers the fabrication cost. In addition, the coupler proposed here shows a wide-band character with a 1-dB bandwidth of $64 \mathrm{~nm}$ and 3-dB bandwidth of $96 \mathrm{~nm}$. Such a grating coupler design can provide an efficient and cost-effective solution for vertical fiber-to-chip optical coupling of a Wavelength Division Multiplexing (WDM) application.
\end{abstract}

Keywords: silicon photonics; grating coupler; backside metal mirror; photonic integrated circuit

\section{Introduction}

Silicon photonics has enabled a wide range of applications ranging from light-based communication to interconnect to low-cost lab-on-a-chip systems [1]. Benefitting from the mature complementary metal-oxide-semiconductor (CMOS) technology, various photonic devices have been demonstrated base on low loss silicon-on-insulator (SOI) waveguide, showing great promise for electronic-photonic integrated circuits, high-density photonic integrated circuits (PICs), and three-dimensional (3D) photonic integration [2-6]. However, since there is no mature silicon-based light source, a fiber coupler is still needed to couple off-chip light from a single mode fiber (SMF) into a submicron SOI waveguide in a PIC [7]. Inverse tapers and grating couplers are the two most commonly used interfaces between a silicon PIC and SMF. High coupling efficiency (CE) and wide bandwidth can be achieved simultaneously using inverse tapers [8]. However, there are a number of drawbacks using such approaches. Typically, specialized optical fibers and cleaved devices with polished facets are required. Very precise optical alignment is needed, which drastically increases the 
cost. The placement of inverse tapers are quite inconvenient since they have to couple light from the edge of the PIC chip. Grating couplers have been studied extensively and are attractive because they are easier to fabricate, are more flexible in their placement, and they enable to test their performance on the wafer-scale [9].

Conventional grating couplers couple light from tilted fiber to avoid second-order reflection [10,11]. Fiber angle tuning and polishing are always needed in optical tests and packaging of silicon PICs, which are disadvantageous for rapid wafer-scale tests and low-cost photonic packaging $[12,13]$. Therefore, perfectly vertical grating couplers with high CE would be very attractive as they can further lower the cost of photonic packaging.

Many designs have been proposed to achieve perfectly vertical couplers, such as a slanted grating coupler, chirped grating coupler, a coupler with extra reflector, and dual-layer grating coupler. A slanted grating coupler breaks the symmetry problem associated with the surface normal coupling between fiber and the planar waveguide, resulting in higher radiation directionality [14]. Chirped grating is a common approach to achieve efficient vertical coupling because chirping the grating structure will introduce asymmetry to the grating coupler [15,16]. Another way to introduce asymmetry to the grating is to add an extra etched reflector $[17,18]$. With the help of the reflector, coupling into unwanted modes could be decreased. As for a dual-layer grating coupler, an inter-layer lateral shift is introduced between the two layers to mimic tilted mirror behavior [19-22], so that high directionality and low back-reflection could be simultaneously realized. However, these approaches require either extra fabrication processes or sophisticated device structure with quite small minimum feature size, which increases the fabrication cost drastically and affects the fabrication error tolerance.

In order to avoid complicated design or extra fabrication steps, a grating coupler that enables vertical to in-plane bidirectional transmission with bi-layer anti-reflection cladding was proposed in our previous work [23]. The bidirectional grating coupler functions as both a fiber coupler and optical power splitter, so that it cannot only act as a highly efficient vertical coupler but also work in Mach-Zehnder type optical modulators [24-26]. However, directly depositing $\mathrm{Si}_{3} \mathrm{~N}_{4}$ on SOI grating introduces extra deposition and etching steps into CMOS technology. In this work, we propose a new design of a bidirectional grating coupler with enhanced efficiency. Perfectly vertical coupling between SMF and SOI waveguide with high efficiency is numerically demonstrated. With the help of an additional $\mathrm{Si}_{3} \mathrm{~N}_{4}$ layer above grating, enhanced $\mathrm{CE}$ is achieved with a cost-effective uniform grating coupler. Since the grating is covered by silicon oxide $\left(\mathrm{SiO}_{2}\right)$, a $\mathrm{Si}_{3} \mathrm{~N}_{4} / \mathrm{SiO}_{2}$ interface is created above the grating. With the help of this interface, maximal coupling into the guided mode and destructive interference in unwanted modes can be achieved. The reflection back into the fiber is reduced and total in-plane CE is increased. Compared with our previous work [23], both $\mathrm{Si}_{3} \mathrm{~N}_{4} / \mathrm{SiO}_{2}$ structures are above the grating to reduce the back-reflection in both designs, but in a different manner. In our previous work, the $\mathrm{Si}_{3} \mathrm{~N}_{4}$ layer was sandwiched by the grating and silicon oxide, while in this new design, the $\mathrm{Si}_{3} \mathrm{~N}_{4}$ layer is above the silicon oxide. The change of the order makes the proposed device more fabrication-compatible with CMOS technology, since $\mathrm{Si}_{3} \mathrm{~N}_{4}$ is a commonly used dielectric as the passivation layer in back end of line in the CMOS process. We believe that such a design can provide an efficient and cost-effective solution for vertical coupling applications and low-cost fiber packaging for silicon PICs.

\section{Device Structure and Principle}

A schematic of the vertical grating coupler is shown in Figure 1. As can be seen clearly, the device we propose is based on a uniform grating with two opposite in-plane transmission ports. The core structure of the device is a uniform grating with period of $\Lambda$. Compared with some sophisticated designs, such as chirped grating couplers [18,22], uniform grating couplers have a fairly larger minimum feature size about half of the wavelength in the SOI waveguide. This larger minimum feature size ensures that the proposed coupler is a cost-effective device. 
When a perfectly vertical SMF is placed in the center of the uniform grating, transversal electric (TE) polarized light from the fiber is diffracted by the grating structure and coupled into two waveguide modes with opposite directions. According to the Bragg condition, the perfectly vertical coupling can be achieved with a carefully designed grating parameter. The total CE for perfectly vertical in-plane coupling is mainly affected by up-reflection and substrate leakage. The up-reflection power attracts more concern since it will not only decrease the CE but also lead to light source degradation because of the power reflected back through fiber.

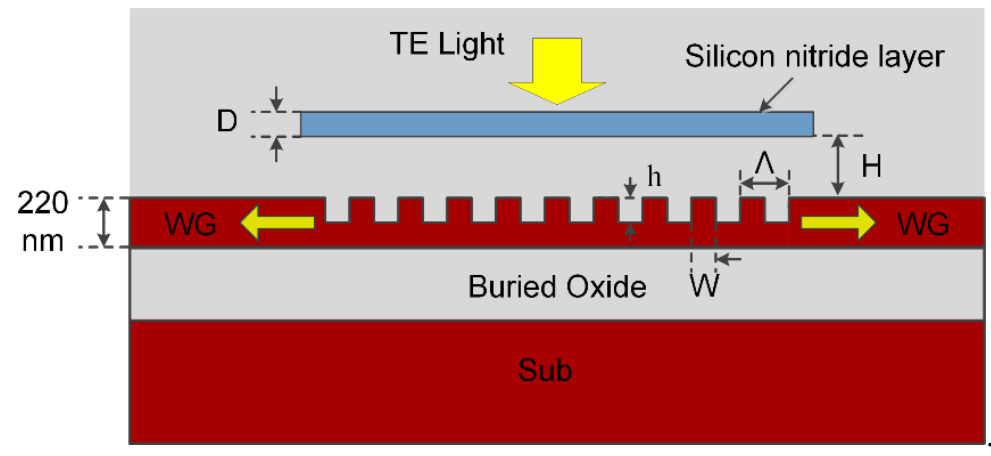

Figure 1. Schematic diagram of the device configuration.

In the current design we add a specific $\mathrm{Si}_{3} \mathrm{~N}_{4}$ layer with carefully designed thickness $D$ and gap to grating $H$ to reduce the up-reflection power. By introducing the $\mathrm{Si}_{3} \mathrm{~N}_{4}$ layer, a $\mathrm{Si}_{3} \mathrm{~N}_{4} / \mathrm{SiO}_{2}$ interface is created above the grating. Considering the silicon $/ \mathrm{SiO}_{2}$ interface introduced by grating itself, the whole structure can be seen as a Fabry-Pérot interferometer. Thus, the reduction of up-reflection can be explained with the help of the Fabry-Pérot interferometer; constructive interference occurred in guide mode and destructive interference in up-reflected mode.

\section{Design and Simulation}

\subsection{Design of a Vertical Grating Coupler with $\mathrm{Si}_{3} \mathrm{~N}_{4}$ Layer}

We started with the design of a vertical grating coupler without an additional $\mathrm{Si}_{3} \mathrm{~N}_{4}$ layer. The coupler is based on a uniform grating where the first-order diffraction couples light out of the waveguide, producing a surface-normal propagating field [27]. According to the Bragg condition, we can achieve a $90^{\circ}$ coupling with a grating when the grating period $\Lambda$ equals the wavelength divided

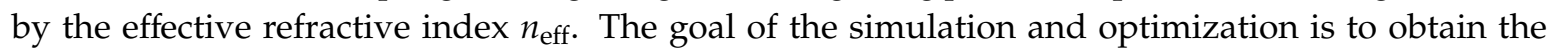
highest average CE in C band (1530 1565 nm). The whole simulation was done using two-dimensional finite-difference time-domain (FDTD) simulations with commercial software Lumerical FDTD Solutions (Lumerical Inc., Vancouver, Canada). The device was designed to be fabricated on a SOI substrate with 220-nm-thick top silicon and 2 - $\mu$ m-thick buried oxide. The grating groove depth $h$ was chosen as $70 \mathrm{~nm}$, a measurement widely used in most silicon photonic fabrication platforms. The grating period number was 17 and the refractive index of $\mathrm{Si}_{3} \mathrm{~N}_{4}$ was 2.03 [28].

Grating is a polarization sensitive device and TE polarized light is of great interest in most cases of silicon PICs. Therefore, the input SMF mode was modeled using a TE polarized Gaussian source with mode waist of $5.2 \mu \mathrm{m}$. The average $C E$ in $C$ band with different $\Lambda$ and filling factor $(F F=W / \Lambda$, where $W$ is the grating teeth width) are shown in Figure $2 a$, where $\Lambda=575 \mathrm{~nm}$ and FF $=0.49$ were chosen as the optimal design parameters for the vertical grating coupler with an average $\mathrm{CE}$ in $\mathrm{C}$ band of about $56.3 \%$. The up-reflection and substrate leakage of the simple vertical grating coupler using these optimal parameters was then calculated and plotted in Figure 2b. As shown clearly, nearly half of the input light power is either coupled to the substrate or reflected back to fiber. The average up-reflection power in $\mathrm{C}$ band is so high that the return loss is reaching $-7.5 \mathrm{~dB}$. High return loss will induce damage to the light source, which is unacceptable in a practical system. 
To reduce the up-reflection, we then added an additional $\mathrm{Si}_{3} \mathrm{~N}_{4}$ layer above the grating region. As discussed above, the $\mathrm{Si}_{3} \mathrm{~N}_{4}$ layer introduced a $\mathrm{Si}_{3} \mathrm{~N}_{4} / \mathrm{SiO}_{2}$ interface that plays a key role in the CE enhancement. The gap $H$ between $\mathrm{Si}_{3} \mathrm{~N}_{4}$ layer and grating has a major influence on the CE. It should be note that the incident wave will be partially reflected by the grating, which is the up-reflection wave. In the presence of $\mathrm{Si}_{3} \mathrm{~N}_{4}$ layer, the up-reflected wave partially reflects back towards the grating at the $\mathrm{Si}_{3} \mathrm{~N}_{4} / \mathrm{SiO}_{2}$ interface, as illustrated in Figure 3. The gap $H$ should be chosen such that the waves reflected back by the $\mathrm{Si}_{3} \mathrm{~N}_{4} / \mathrm{SiO}_{2}$ interface interfere constructively with the wave coupled into the grating. To ensure the constructive interference, the reflected wave and incident wave should be in phase. As depicted in Figure 3, the phase difference between the incident wave and reflected wave is:

$$
\Delta \varphi=2 \pi \frac{2 H}{\lambda_{0} / n_{O X}}+\varphi_{r}+\varphi_{\text {Grating }}
$$

where $\lambda_{0}$ is the wavelength of the input light, $n_{\mathrm{OX}}$ is the refractive index of $\mathrm{SiO}_{2}, \varphi_{r}$ is the phase shift at the $\mathrm{Si}_{3} \mathrm{~N}_{4} / \mathrm{SiO}_{2}$ interface, which is $\pi$, and $\varphi_{\text {Grating }}$ is the phase shift at the grating/SiO $\mathrm{O}_{2}$ interface. When $\Delta \varphi$ equals $2 m \pi$ ( $m$ is an integer), constructive interference occurs and hence coupling into the grating will be enhanced.

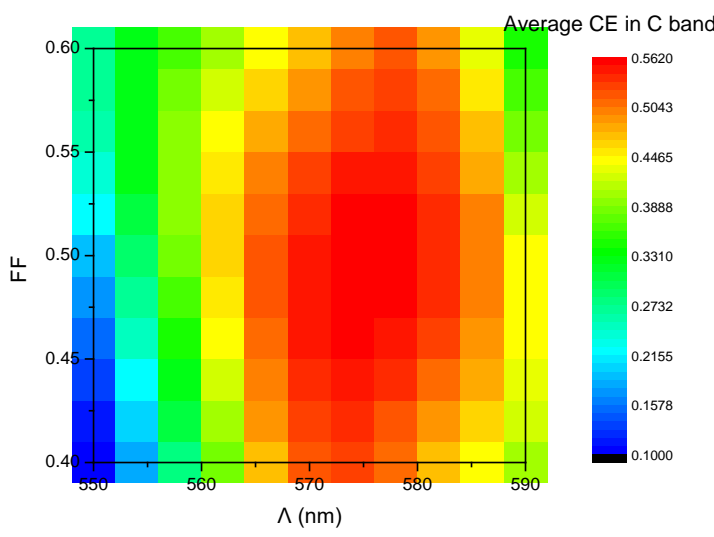

(a)

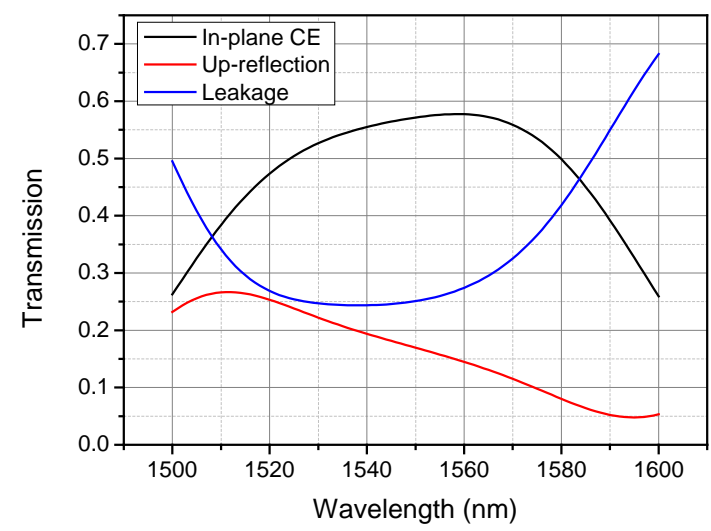

(b)

Figure 2. (a) The average coupling efficiency (CE) in $C$ band with different $\Lambda$ and FF. The grating coupler with $\Lambda=575 \mathrm{~nm}$ and filling factor $(\mathrm{FF})=0.49$ achieves an average $\mathrm{CE}$ in $\mathrm{C}$ band of about $56.3 \%$. (b) Calculated transmission spectra of the simple vertical grating coupler with $\Lambda=575 \mathrm{~nm}$ and $\mathrm{FF}=0.49$.

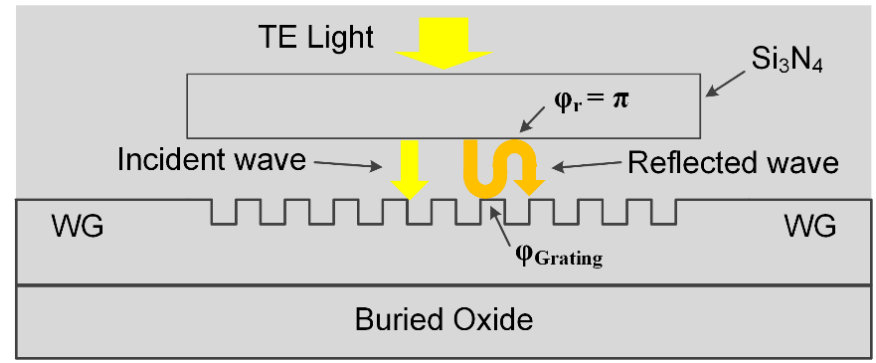

Figure 3. Schematic of the phase difference between incident wave and reflected wave.

For simplicity, we assume that $\varphi_{\text {Grating }}=\pi$, since the effective refractive index of the grating is higher than $n_{O X}$. Therefore, constructive interference occurs when $H=m \lambda_{0} /\left(2 n_{O X}\right)$. Considering $\lambda_{0}$ $=1550 \mathrm{~nm}, n_{O X}$ is 1.45 , and $m$ is 1 , then the gap $H$ is calculated as $530 \mathrm{~nm}$. With this calculated gap, the thickness of the $\mathrm{Si}_{3} \mathrm{~N}_{4}$ layer $D$ is determined by a parameter sweep through a series of numerical simulations, as shown in Figure $4 \mathrm{a}$. The parameters of the grating are the same as the vertical grating coupler designed above. As shown in Figure $4 \mathrm{a}$, the CE is a periodical function of the thickness $D$. 
By choosing $D$ properly to add destructive interference between the wave reflected by grating and the wave reflected by $\mathrm{Si}_{3} \mathrm{~N}_{4}$ layer, the back-reflection is diminished so that the coupling into guided mode is enhanced. From Figure $4 \mathrm{a}, D$ is set to be $930 \mathrm{~nm}$ to achieve the highest average CE in $\mathrm{C}$ band of about $67.2 \%$; the waves are reflected by grating and reflected by $\mathrm{Si}_{3} \mathrm{~N}_{4}$ interfere at the upper $\mathrm{Si}_{3} \mathrm{~N}_{4} / \mathrm{SiO}_{2}$ interface. The up-reflection and substrate leakage of the efficiency enhanced vertical grating coupler with $H=530 \mathrm{~nm}$ and $D=930 \mathrm{~nm}$ are then calculated and plotted in Figure $4 \mathrm{~b}$. As shown clearly, the peak $\mathrm{CE}$ is about $68.7 \%(-1.63 \mathrm{~dB})$ at $1549 \mathrm{~nm}$, and $1-\mathrm{dB}$ and $3-\mathrm{dB}$ bandwidths are about $56 \mathrm{~nm}$ and $85 \mathrm{~nm}$, showing a wideband feature. The average up-reflection of the efficiency enhanced coupler is as low as $4.9 \%$, showing $5.6 \mathrm{~dB}$ improvement in return loss compared to the simple vertical grating coupler without $\mathrm{Si}_{3} \mathrm{~N}_{4}$ layer. However, as shown in the blue curve in Figure $4 \mathrm{~b}$, around $25 \%$ of the light power leak into the silicon substrate, which limits the total in-plane CE.

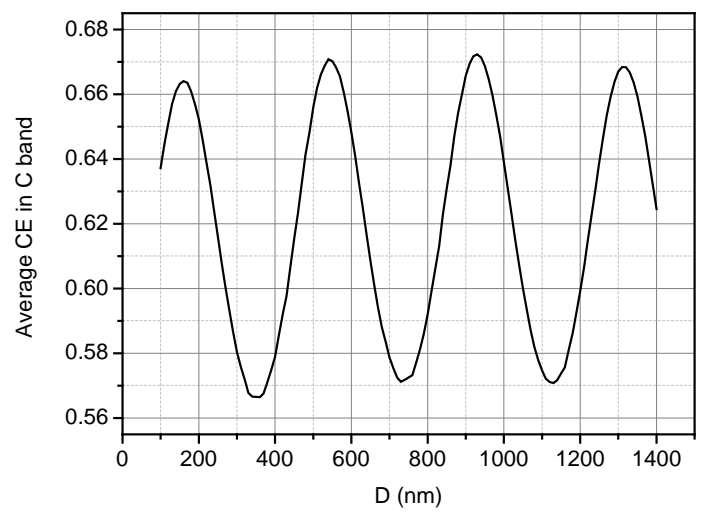

(a)

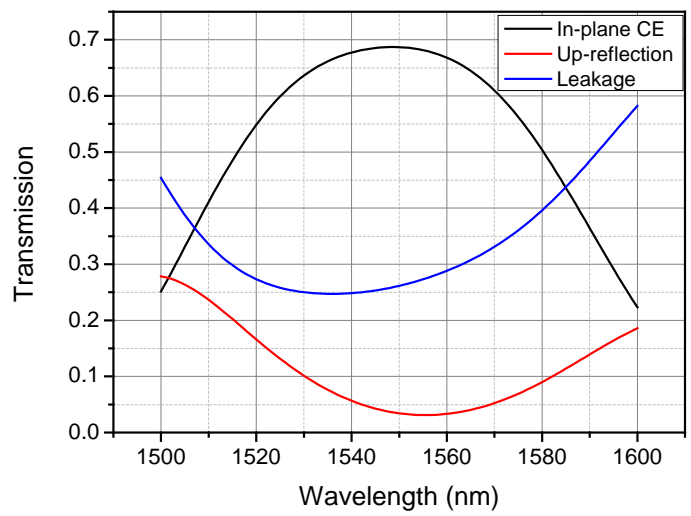

(b)

Figure 4. (a) Average CE in $C$ band with different $D$; (b) calculated transmission spectra of vertical grating coupler with $H=530 \mathrm{~nm}$ and $D=930 \mathrm{~nm}$.

The effect of length of the $\mathrm{Si}_{3} \mathrm{~N}_{4}$ layer on $\mathrm{CE}$ is analyzed through a parameter sweep. Figure 5 shows that the length of the $\mathrm{Si}_{3} \mathrm{~N}_{4}$ layer will only affect the coupling seriously if it is smaller than the length of grating. There is a peak CE when the length is about the same as the grating length shown in Figure 5. However, as long as the $\mathrm{Si}_{3} \mathrm{~N}_{4}$ layer is longer than the grating, the coupling efficiency will not vary with the length of the $\mathrm{Si}_{3} \mathrm{~N}_{4}$ layer. The peak $\mathrm{CE}$ is believed to be the result of edge diffraction of the $\mathrm{Si}_{3} \mathrm{~N}_{4}$ layer. However, the peak CE is about only 0.015 higher than the CE when the $\mathrm{Si}_{3} \mathrm{~N}_{4}$ layer is longer than the grating. Therefore, it is possible to deposit a full $\mathrm{Si}_{3} \mathrm{~N}_{4}$ layer to avoid an extra etching process. Directly using the $\mathrm{Si}_{3} \mathrm{~N}_{4}$ as the passivation layer over the $\mathrm{SiO}_{2}$ layer is also possible since the UV glue or index matching fluid will be used between the fiber and the photonic chip in most cases.

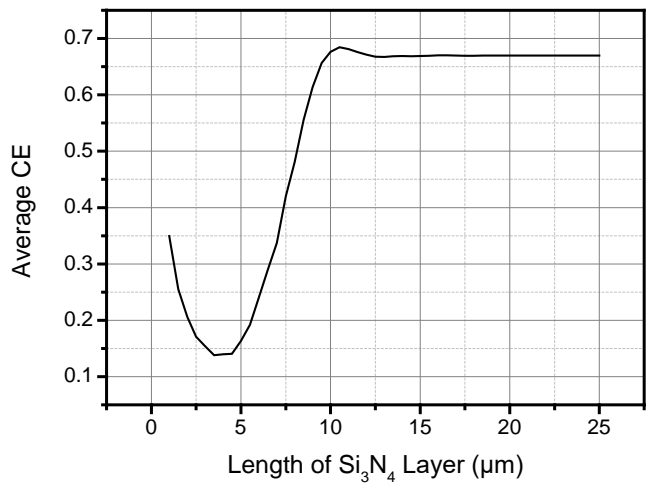

Figure 5. Average in-plane $C E$ in $C$ band with different lengths of $\mathrm{Si}_{3} \mathrm{~N}_{4}$ layer. As long as the $\mathrm{Si}_{3} \mathrm{~N}_{4}$ layer is longer than the grating, the coupling efficiency will not vary with the length of the $\mathrm{Si}_{3} \mathrm{~N}_{4}$ layer. 
The cross-sectional view of the electric intensity profiles at wavelength of $1550 \mathrm{~nm}$ was calculated for grating coupler without and with an additional $\mathrm{Si}_{3} \mathrm{~N}_{4}$ layer respectively, as shown in Figure 6 . From these result plots, we can clearly see the coupling scheme and the power flow in different directions. Obviously, constructive interference occurred between the $\mathrm{Si}_{3} \mathrm{~N}_{4}$ layer and grating, resulting in diminished up-reflection.

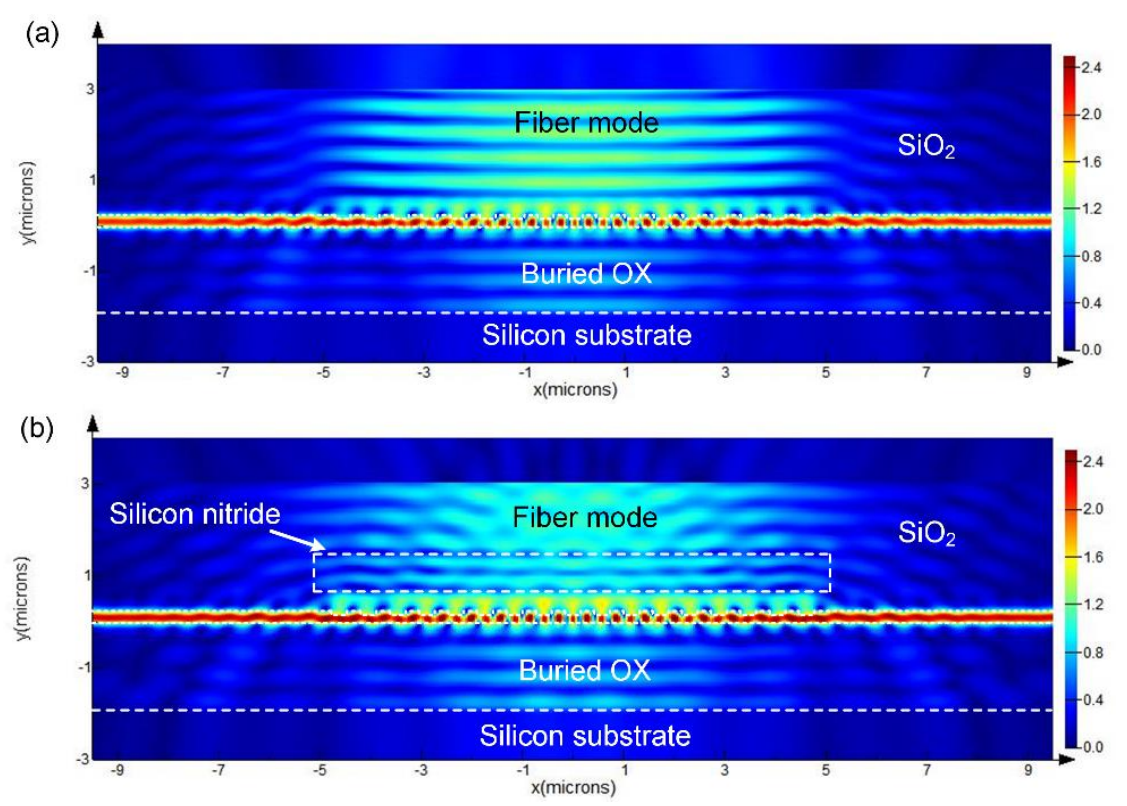

Figure 6. The cross-sectional view of the electric intensity profiles at wavelength of $1550 \mathrm{~nm}$ for (a) vertical grating coupler without $\mathrm{Si}_{3} \mathrm{~N}_{4}$ layer and (b) CE enhanced vertical grating coupler with $H=530 \mathrm{~nm}$ and $D=930 \mathrm{~nm}$.

\subsection{Optimization Using Genetic Algorithm (GA)}

As shown in Figure 1, there are five layers in the whole device, namely, silicon substrate, buried oxide, top silicon, covered $\mathrm{SiO}_{2}$ and $\mathrm{Si}_{3} \mathrm{~N}_{4}$; this results in five interfaces existing in the whole coupler. Apparently, all interfaces could have contributed to the reduction of up-reflection. Due to the phase response of the sub-wavelength grating and the effect of all interfaces, the phase-matching condition is a little complicated, and accurate analytical calculation of the thickness $D$ and gap $H$ is quite difficult. Moreover, when considering the grating and $\mathrm{Si}_{3} \mathrm{~N}_{4}$ layer as a whole device, the optimal coupling condition may happen with different $\Lambda, \mathrm{FF}$, and $h$ compared to the vertical grating coupler designed above. Therefore, in order to achieve good coupling performance, all the five parameters $(D, H$, $\Lambda, \mathrm{FF}$ and $h$ ) should be carefully designed, which is a typical multi-peak problem that needs a lot of calculation.

Fortunately, the two-dimensional FDTD simulation of the proposed device is not time-consuming, so that we can turn to GA to help find the optimal design parameter. GA is a global search algorithm which applies the principle of survival of the fittest. It does not depend on the gradient information when optimizing the calculation and does not require the objective function to be continuous and steerable, making it suitable to optimize the parameters of a grating coupler. Moreover, it has already proven helpful in related fields $[11,16,29,30]$.

Here we set the average $\mathrm{CE}$ in $\mathrm{C}$ band as the figure of merit (FOM). Simulation results of the final optimized design obtained through GA are shown in Figure 6. The FOM trend with the iterative process is shown in Figure 7a. As can be seen, the simulation reaches convergence after the 600th generation. The optimal parameter of the whole coupler is $\Lambda=579 \mathrm{~nm}, \mathrm{FF}=0.46, D=959 \mathrm{~nm}$, $H=498 \mathrm{~nm}$, and $h=75 \mathrm{~nm}$. The GA-optimized coupler is capable of achieving average CE in C band of $68.5 \%$ and the peak CE of $71 \%$ occurred in $1553 \mathrm{~nm}$ with a 1-dB bandwidth of $53 \mathrm{~nm}$ and 3-dB 
bandwidth of $85 \mathrm{~nm}$, as shown in the transmission spectra. Compared with the coupler designed in Section 3.1., the average up-reflection in C band of the GA-optimized coupler is increased slightly to $7.4 \%$ as shown in Figure $7 \mathrm{~b}$.

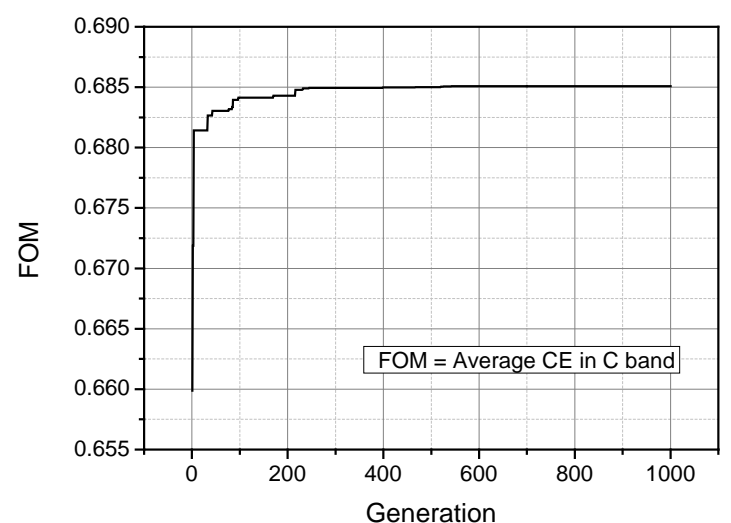

(a)

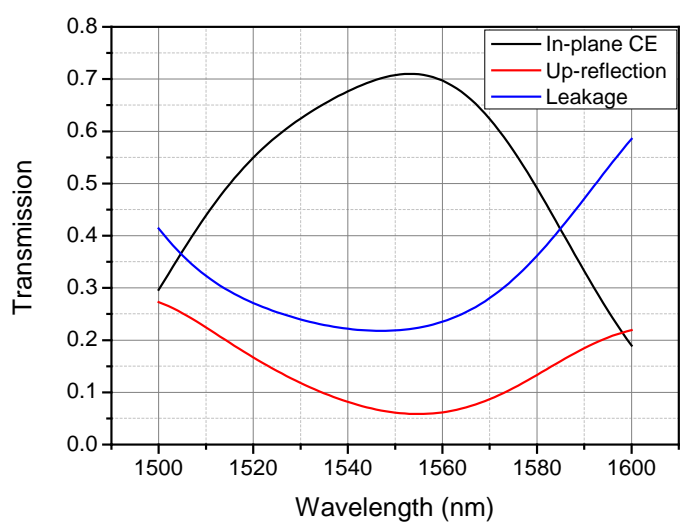

(b)

Figure 7. Results for genetic algorithm (GA)-optimized coupler (a) runtime statistics of maximum and average CE; (b) calculated transmission spectra for the final optimal design with $\Lambda=579 \mathrm{~nm}, \mathrm{FF}=0.46$, $D=959 \mathrm{~nm}, H=498 \mathrm{~nm}$, and $h=75 \mathrm{~nm}$.

Obviously, the optimal $H$ and $D$ obtained through GA are a little different from the calculated $H$ and $D$ in Section 3.1. This difference occurs because of the simplification we made that $\varphi_{\text {Grating }}=\pi$. Apparently, phase shift of the grating is more complicated because of effects of all interfaces in whole grating. However, the difference between the calculated CE in Section 3.1. and the GA-optimized design is quite small, which indicates that the proposed device is a fabrication-tolerant design. Moreover, the minimum line width of the proposed coupler is $266 \mathrm{~nm}$, which can be easily fabricated with $193 \mathrm{~nm}$ deep-UV lithography and lowers the fabrication cost.

\subsection{Combined Coupler and Backside Metal Mirror}

To further increase the efficiency of grating couplers, the directionality of the grating should be optimized since there are still about more than $20 \%$ incident power couple into silicon substrate at $1550 \mathrm{~nm}$ in our design. A backside metal mirror has proven to be effective in redirecting the optical power diffracted to the substrate, leading to a high CE [31-33]. In addition, the backside metal mirror can be realized with CMOS compatible processes such as deep-UV photolithography, etching, and metal deposition, thus simplifying the integration of a metal mirror with grating coupler [33,34]. In our simulation, a 60-nm-thick aluminum layer was placed under the buried oxide layer to act as a backside metal mirror. As depicted in Figure 8, an average CE of $87 \%$ and peak CE of $89.4 \%$ were achieved, which show an appreciable improvement of around $1.05 \mathrm{~dB}$ in the average CE. Since the diffracted power penetrating the grating is reflected at the metal mirror, an important part is redirected to the grating and coupled into guided mode, resulting in enhanced efficiency of the grating coupler. Moreover, a 1-dB bandwidth amelioration from 53 to $64 \mathrm{~nm}$ and a 3-dB bandwidth amelioration from 85 to $96 \mathrm{~nm}$ are achievable. 


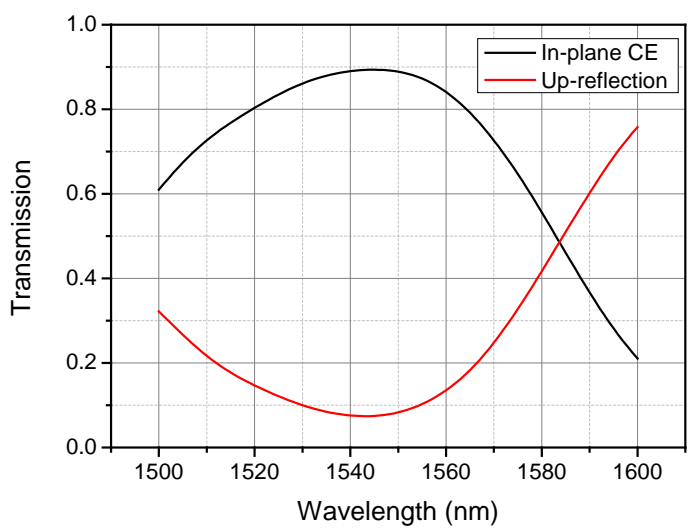

Figure 8. Calculated transmission spectra for the GA-optimized design with a 60-nm-thick aluminum backside metal mirror, showing increased CE and bandwidth.

\section{Discussion}

As mentioned in Section 3.1., the grating period of the proposed device is about $\lambda_{0} / n_{\text {eff }}$, and the second-order back-reflection is of great concern. In the case of coupling from chip to fiber, lights will be incident to the grating region from two waveguides on both sides of the proposed bidirectional grating. While for a conventional grating coupler connected to a single waveguide, light is incident from one waveguide. Therefore, the second-order back-reflection will be of great concern when the period of the conventional grating coupler is around $\lambda_{0} / n_{\text {eff }}$.

The back-reflection is calculated using power monitors positioned behind mode sources in the two-dimensional (2D) FDTD simulation. The CE into fiber is calculated by overlap integral between the fiber mode and the field from the power monitor over the grating. The simulation set-up with two mode sources is depicted in Figure 9, and all parameters of the grating are the same as the ones in Section 3.3. Firstly, simulation with one mode source positioned at the left waveguide is carried out to analyze the performance of a conventional grating coupler. As shown in Figure 10a,b, the back-reflection reaches as high as $31 \%$ at $1535 \mathrm{~nm}$ and about $18 \%$ power of light transmit through the grating. However, for the bidirectional grating, since there are guided waves propagating to the grating region from both sides, the wave reflected back into the right waveguide interferes destructively with the wave transmitting through the grating from the left waveguide. The same happens to the wave reflected into the left waveguide. Destructive interference between the reflected wave and transmitted wave results in diminished back-reflection into waveguides of about $1.5 \%$; the simulation results are shown in Figure 10c,d. The CE from waveguides to fiber matches well with the case fiber-to-chip coupling.

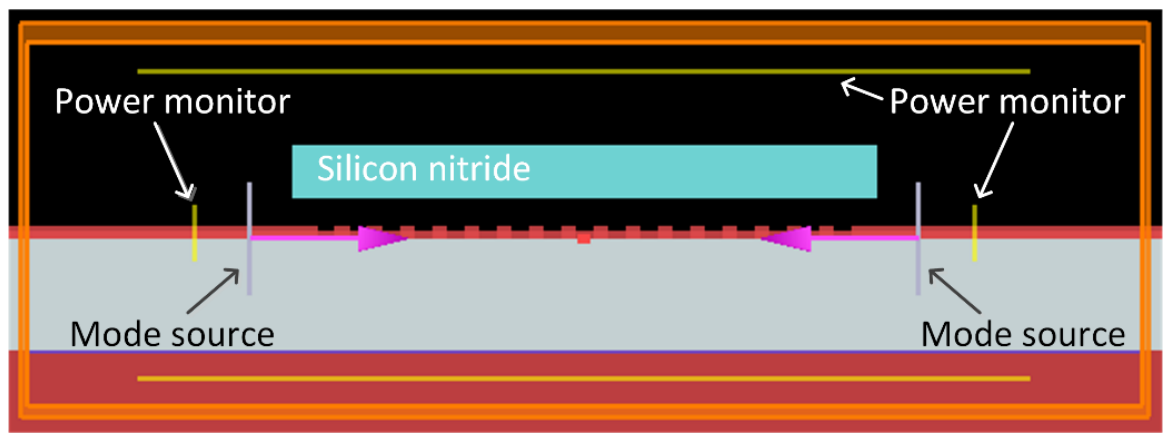

Figure 9. The simulation set-up in the case of chip-to-fiber coupling for the bidirectional grating. Power monitors are set behind mode sources on both sides of grating to calculate back-reflection. 
(a)

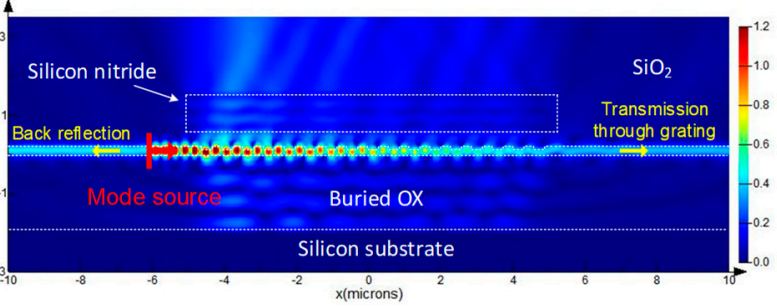

(c)

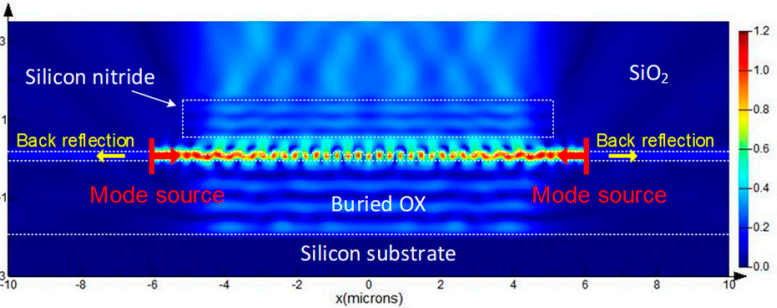

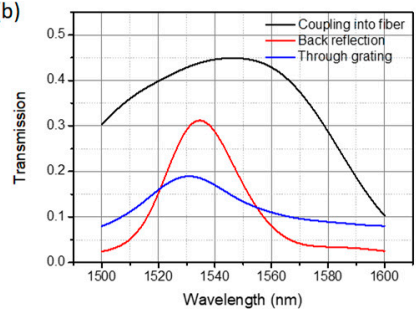

(d)

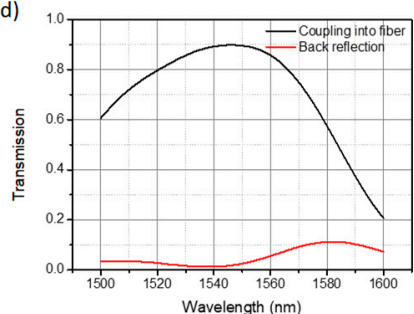

Figure 10. Two-dimensional (2D) finite-difference time-domain (FDTD) simulation results for grating coupler with one mode source positioned at left waveguide: (a) cross-sectional view of the electric intensity profile at wavelength of $1550 \mathrm{~nm}$; (b) waveguide-to-fiber CE, second-order back-reflection into waveguide and transmission through grating. 2D FDTD simulation results for bidirectional grating coupler with two mode sources positioned on both sides: (c) cross-sectional view of the electric intensity profile at wavelength of $1550 \mathrm{~nm}$; (d) waveguide-to-fiber CE and back-reflection into waveguide.

\section{Conclusions}

In conclusion, a high efficiency and broadband vertical grating coupler is designed and numerically demonstrated. Assisted by a $\mathrm{Si}_{3} \mathrm{~N}_{4}$ layer above the grating, the total in-plane $\mathrm{CE}$ of the vertical grating coupler is enhanced greatly while up-reflection is diminished. Genetic algorithm is employed to simultaneously optimize the grating and $\mathrm{Si}_{3} \mathrm{~N}_{4}$ layer. The average $\mathrm{CE}$ and up-reflection in $\mathrm{C}$ band of GA-optimized coupler are $68.5 \%(-1.65 \mathrm{~dB})$ and $7.4 \%(-11.3 \mathrm{~dB})$, respectively. With the help of a backside metal mirror, the average $\mathrm{CE}$ in $\mathrm{C}$ band and peak $\mathrm{CE}$ are further increased to $87 \%(-0.6 \mathrm{~dB})$ and $89.4 \%(-0.49 \mathrm{~dB})$, respectively. The minimum feature size of the designed device is $266 \mathrm{~nm}$, which makes our design easy to fabricate through $193 \mathrm{~nm}$ DUV lithography. With a large optical bandwidth (1-dB bandwidth of $64 \mathrm{~nm}, 3-\mathrm{dB}$ bandwidth of $96 \mathrm{~nm}$ ) and flat-top filtering characteristic, this vertical coupler is believed to be suitable for the optical interface of a WDM application and low-cost fiber packaging for silicon PICs.

Author Contributions: Conceptualization, Z.Z. (Zan Zhang); simulation, Z.Z. (Zan Zhang) and X.S.; results, analysis, and discussion, B.H., B.B., Z.Z. (Zanyun Zhang), C.C., and T.G.; writing-original draft preparation, Z.Z. (Zan Zhang) and X.S.; writing - review and editing, X.X., L.Z., and H.C.; supervision, H.C. All authors have read and agreed to the published version of the manuscript.

Funding: This research was funded by the National Key R\&D Program of China (Grant Nos. 2018YFA0209000, 2017YFA0205903), the Natural Science Foundation of China (Grant Nos. 61704009, 61675191, 61974099, 61634006, and 61805020), the National Natural Science foundation of Shaanxi Province, China (Grant No. 2018JZ6004), and the Fundamental Research Funds for the Central Universities, CHD (Grant No. 300102320106).

Conflicts of Interest: The authors declare no conflict of interest.

\section{References}

1. Soref, R. The Past, Present, and Future of Silicon Photonics. IEEE J. Sel. Top. Quantum Electron. 2006, 12, 1678-1687. [CrossRef]

2. Miller, D.A.B. Device Requirements for Optical Interconnects to Silicon Chips. Proc. IEEE 2009, 97, 1166-1185. [CrossRef]

3. Brimont, A.; Thomson, D.J.; Gardes, F.Y.; Fedeli, J.M.; Reed, G.T.; Martí, J.; Sanchis, P. High-contrast 40 Gb/s operation of a $500 \mu \mathrm{m}$ long silicon carrier-depletion slow wave modulator. Opt. Lett. 2012, 37, 3504-3506. [CrossRef] 
4. Zhang, Z.; Li, H.; Huang, B.; Zhang, Z.; Cheng, C.; Gao, T.; Yu, Y.; Li, Y.; Chen, H. Multi-channel silicon photonic receiver based on compact second-order microring resonators. Opt. Commun. 2019, 437, 168-173. [CrossRef]

5. Madani, A.; Kleinert, M.; Stolarek, D.; Zimmermann, L.; Ma, L.; Schmidt, O.G. Vertical optical ring resonators fully integrated with nanophotonic waveguides on silicon-on-insulator substrates. Opt. Lett. 2015, 40, 3826-3829. [CrossRef] [PubMed]

6. Chin, M.-K.; Lee, C.-W.; Shen, J. Polarization-independent vertical coupler for photonics integration. Opt. Express 2004, 12, 117-123. [CrossRef] [PubMed]

7. Kopp, C.; Bernabé, S.; Bakir, B.B.; Fedeli, J.; Orobtchouk, R.; Schrank, F.; Porte, H.; Zimmermann, L.; Tekin, T. Silicon Photonic Circuits: On-CMOS Integration, Fiber Optical Coupling, and Packaging. IEEE J. Sel. Top. Quantum Electron. 2011, 17, 498-509. [CrossRef]

8. Almeida, V.R.; Panepucci, R.R.; Lipson, M. Nanotaper for compact mode conversion. Opt. Lett. 2003, 28, 1302-1304. [CrossRef]

9. Roelkens, G.; Vermeulen, D.; Selvaraja, S.; Halir, R.; Bogaerts, W.; Van Thourhout, D. Grating-Based Optical Fiber Interfaces for Silicon-on-Insulator Photonic Integrated Circuits. IEEE J. Sel. Top. Quantum Electron. 2011, 17, 571-580. [CrossRef]

10. Chen, X.; Cheng, Z.; Fung, C.K.Y.; Tsang, H.K. Design and applications of silicon waveguide grating couplers. In Proceedings of the Silicon Photonics VII, International Society for Optics and Photonics, San Francisco, CA, USA, 2 February 2012; Volume 8266, p. 82660I.

11. Roelkens, G.; Thourhout, D.V.; Baets, R. High efficiency Silicon-on-Insulator grating coupler based on a poly-Silicon overlay. Opt. Express 2006, 14, 11622-11630. [CrossRef]

12. Marchetti, R.; Lacava, C.; Carroll, L.; Gradkowski, K.; Minzioni, P. Coupling strategies for silicon photonics integrated chips [Invited]. Photonics Res. 2019, 7, 201-239. [CrossRef]

13. Nambiar, S.; Sethi, P.; Selvaraja, S.K. Grating-Assisted Fiber to Chip Coupling for SOI Photonic Circuits. Appl. Sci. 2018, 8, 1142. [CrossRef]

14. Wang, B.; Jiang, J.; Nordin, G.P. Embedded slanted grating for vertical coupling between fibers and silicon-on-insulator planar waveguides. IEEE Photonics Technol. Lett. 2005, 17, 1884-1886. [CrossRef]

15. Chen, X.; Li, C.; Tsang, H.K. Fabrication-Tolerant Waveguide Chirped Grating Coupler for Coupling to a Perfectly Vertical Optical Fiber. IEEE Photonics Technol. Lett. 2008, 20, 1914-1916. [CrossRef]

16. Tong, Y.; Zhou, W.; Tsang, H.K. Efficient perfectly vertical grating coupler for multi-core fibers fabricated with 193 nm DUV lithography. Opt. Lett. 2018, 43, 5709-5712. [CrossRef]

17. Taillaert, D.; Bogaerts, W.; Bienstman, P.; Krauss, T.F.; Van Daele, P.; Moerman, I.; Verstuyft, S.; De Mesel, K.; Baets, R. An out-of-plane grating coupler for efficient butt-coupling between compact planar waveguides and single-mode fibers. IEEE J. Quantum Electron. 2002, 38, 949-955. [CrossRef]

18. Roelkens, G.; Thourhout, D.V.; Baets, R. High efficiency grating coupler between silicon-on-insulator waveguides and perfectly vertical optical fibers. Opt. Lett. 2007, 32, 1495-1497. [CrossRef]

19. Dai, M.; Ma, L.; Xu, Y.; Lu, M.; Liu, X.; Chen, Y. Highly efficient and perfectly vertical chip-to-fiber dual-layer grating coupler. Opt. Express 2015, 23, 1691-1698. [CrossRef]

20. Watanabe, T.; Ayata, M.; Koch, U.; Fedoryshyn, Y.; Leuthold, J. Perpendicular Grating Coupler Based on a Blazed Antiback-Reflection Structure. J. Light. Technol. 2017, 35, 4663-4669. [CrossRef]

21. Benedikovic, D.; Alonso-Ramos, C.; Pérez-Galacho, D.; Guerber, S.; Vakarin, V.; Marcaud, G.; Le Roux, X.; Cassan, E.; Marris-Morini, D.; Cheben, P.; et al. L-shaped fiber-chip grating couplers with high directionality and low reflectivity fabricated with deep-UV lithography. Opt. Lett. 2017, 42, 3439. [CrossRef]

22. Michaels, A.; Yablonovitch, E. Inverse design of near unity efficiency perfectly vertical grating couplers. Opt. Express 2018, 26, 4766-4779. [CrossRef]

23. Zhang, Z.; Huang, B.; Zhang, Z.; Cheng, C.; Liu, H.; Li, H.; Chen, H. Highly efficient vertical fiber interfacing grating coupler with bilayer anti-reflection cladding and backside metal mirror. Opt. Laser Technol. 2017, 90, 136-143. [CrossRef]

24. Li, H.; Ma, X.; Cui, B.; Wang, Y.; Zhang, C.; Zhao, J.; Zhang, Z.; Tang, C.; Li, E. Chip-scale demonstration of hybrid III-V/silicon photonic integration for an FBG interrogator. Optica 2017, 4, 692-700. [CrossRef]

25. Huang, B.; Zhang, Z.; Zhang, Z.; Cheng, C.; Zhang, H.; Zhang, H.; Chen, H. 100 Gb/s Silicon Photonic WDM Transmitter with Misalignment-Tolerant Surface-Normal Optical Interfaces. Micromachines 2019, 10, 336. [CrossRef] [PubMed] 
26. Zhang, Z.; Huang, B.; Zhang, Z.; Cheng, C.; Liu, H.; Li, H.; Chen, H. Integrated silicon photonic interconnect with surface-normal optical interface. Opt. Commun. 2016, 367, 206-213. [CrossRef]

27. Hardy, A.; Welch, D.F.; Streifer, W. Analysis of second-order gratings. IEEE J. Quantum Electron. 1989, 25, 2096-2105. [CrossRef]

28. Xiao, X.; Yang, B.; Chu, T.; Yu, J.-Z.; Yu, Y.-D.; Anastasia, N. Design and Characterization of a Top Cladding for Silicon-on-Insulator Grating Coupler. Chin. Phys. Lett. 2012, 29, 114213. [CrossRef]

29. Lin, A.; Phillips, J. Optimization of random diffraction gratings in thin-film solar cells using genetic algorithms. Sol. Energy Mater. Sol. Cells 2008, 92, 1689-1696. [CrossRef]

30. Ahmad, S.U.; Bergen, S.W.A. A genetic algorithm approach to the inverse problem of treatment planning for intensity-modulated radiotherapy. Biomed. Signal Process. Control. 2010, 5, 189-195. [CrossRef]

31. Hoppe, N.; Zaoui, W.S.; Rathgeber, L.; Wang, Y.; Klenk, R.H.; Vogel, W.; Kaschel, M.; Portalupi, S.L.; Burghartz, J.; Berroth, M. Ultra-Efficient Silicon-on-Insulator Grating Couplers With Backside Metal Mirrors. IEEE J. Sel. Top. Quantum Electron. 2020, 26, 1-6. [CrossRef]

32. Kopp, C.; Augendre, E.; Orobtchouk, R.; Lemonnier, O.; Fedeli, J.-M. Enhanced Fiber Grating Coupler Integrated by Wafer-to-Wafer Bonding. J. Light. Technol. 2011, 29, 1847-1851. [CrossRef]

33. Zaoui, W.S.; Rosa, M.F.; Vogel, W.; Berroth, M.; Butschke, J.; Letzkus, F. Cost-effective CMOS-compatible grating couplers with backside metal mirror and 69\% coupling efficiency. Opt. Express 2012, 20, B238-B243. [CrossRef] [PubMed]

34. Stojanović, V.; Ram, R.J.; Popović, M.; Lin, S.; Moazeni, S.; Wade, M.; Sun, C.; Alloatti, L.; Atabaki, A.; Pavanello, F.; et al. Monolithic silicon-photonic platforms in state-of-the-art CMOS SOI processes [Invited]. Opt. Express 2018, 26, 13106-13121. [CrossRef] [PubMed]

(C) 2020 by the authors. Licensee MDPI, Basel, Switzerland. This article is an open access article distributed under the terms and conditions of the Creative Commons Attribution (CC BY) license (http://creativecommons.org/licenses/by/4.0/). 